\title{
Stress-Strain Relationships in Yarns Subjected to Rapid Impact Loading: 5. Wave Propagation in Long Textile Yarns Impacted Transversely
}

\author{
Jack C. Smith, Frank L. McCrackin, and Herbert F. Schiefer
}

\begin{abstract}
The behavior of an infinitely long flexible filament after transverse impact is treated theoretically. The filament is assumed to have a tension-strain curve that is always concave downward, and to have no short-time creep or stress-relaxation effects. Under most conditions the impact initiates a variable strain that propagates down the filament between an "elastic wave" front and a "plastic wave" front. A transverse wave, shaped like an inverted $\mathrm{V}$, then travels in the constant-strain region behind the plastic-wave front. Under special conditions the transverse-wave front may propagate faster than the plastic-wave front, but the shape of the transverse wave remains the same. The theory for both cases is worked out in detail, and some illustrative examples are given.
\end{abstract}

\section{Introduction}

The behavior of textile yarns held in clamps 40 to $60 \mathrm{~cm}$ apart and impacted transversely was demonstrated and discussed in the fourth paper of this series $[1,2,3,4] .{ }^{1}$ The present paper deals with the theory of transverse-wave propagation in yarns of infinite length where there are no clamps to reflect the waves. The theory developed here will be used in future discussions of transverse impact.

When a yarn is struck transversely, a strain wave is propagated along the yarn outward in each direction from the point of impact. In the region between each of these wave fronts, material of the yarn is set into motion longitudinally toward the point of impact. This material is taken up by a tent-shaped wave of transverse motion that propagates outward from the impact point. This behavior is demonstrated in figure 1, in which several configurations of a yarn after transverse impact are shown. The position of the longitudinal-strain wave in each of the configurations is shown by the arrows. The backward flow of yarn material is indicated by the white tick marks.

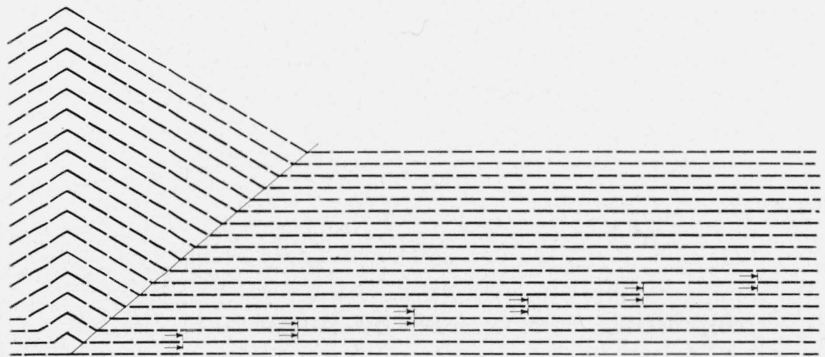

Figure 1. Configurations of a yarn after transverse impact.

These configurations, $40 \times 10^{-6}$ sec apart, were calculated for a yarn having a linear (Hooke's law) tension-strain curve. Longitudinal-strain wave velocity was $2,500 \mathrm{~m} / \mathrm{sec}$. The positions of the longitudinal-wave front in successive configurations, are designated by the arrows. Impact occurred at the vertex of the transverse wave shown at the left, at impact velocity of $180 \mathrm{~m} / \mathrm{sec}$. The leading edge of the transverse wave propagates with velocity $350 \mathrm{~m} / \mathrm{sec}$ with respect to the unstrained yarn material. Inward flow of yarn material at velocity $50 \mathrm{~m} / \mathrm{sec}$ is shown by the white tick marks. There is no horizontal component of flow in the region of the transverse wave.

The configurations shown in figure 1 were calculated for a yarn composed of a Hookean material, using relations developed in this paper. The longitudinal strain-wave velocity was $2,500 \mathrm{~m} / \mathrm{sec}$. Transverse-impact velocity was $180 \mathrm{~m} / \mathrm{sec}$. Under these conditions a 2-percent strain is produced in the wake of the longitudinal-wave front, and yarn material in the region between longitudinal- and transverse-wave fronts flows inward at a velocity of $50 \mathrm{~m} / \mathrm{sec}$. In the wake of the transverse-wave front, which propagates at a velocity of $350 \mathrm{~m} / \mathrm{sec}$, the yarn material flows transversely at the impact velocity as shown. The time interval between each configuration is $40 \times 10^{-6}$ sec.

Transverse waves of the type just described have been discussed by Taylor and his associates [5]. Cole, Dougherty, and Huth [6] have treated the problem of transverse-wave propagation in prestressed wires. Craggs [7] and McCrackin [8] have also discussed the problem for

\footnotetext{
Figures in brackets indicate the literature references at the end of this paper.
} 
plastic-elastic strings, showing the effect of wave reflections and interactions. The problem of oblique impact of an elastic cable has been discussed recently by Ringleb [9]. The treatment presented here extends the work of these authors and makes use of concepts introduced by von Karman [10] and Taylor [11] in their treatments of longitudinal impact.

\section{Theory of Longitudinal Impact}

Longitudinal-strain waves resulting from a constant-velocity tensile impact do not necessarily have sharp wave fronts. According to von Karman, the strain increases gradually in the region between two wave fronts, an "elastic wave" front ${ }^{2}$ propagating at velocity

$$
C_{e}=C(0)=\sqrt{\frac{1}{M}\left(\frac{d T}{d \epsilon}\right)_{\epsilon=0}}
$$

and a "plastic wave" front ${ }^{2}$ propagating more slowly at velocity

$$
C_{p}=C\left(\epsilon_{p}\right)=\sqrt{\frac{1}{M}\left(\frac{d T}{d \epsilon}\right)_{\epsilon=\epsilon_{p}} .}
$$

In eq (1) and (2), $M$ is the mass per unit length of the unstrained filament material, and $d T / d \epsilon$ is the slope of the tension-strain curve, evaluated at zero strain in eq (1) and at strain $\epsilon_{p}$ in eq (2). In the wake of the plastic-wave front the strain in the filament has the constant value $\epsilon_{p}$. The value of $\epsilon_{p}$ depends upon the speed at which the filament is impacted longitudinally.

The filament material in the wake of the plastic-wave front flows in the impact direction at a velocity uniform along the filament given by

$$
W=\int_{0}^{\epsilon_{p}} \sqrt{\frac{1}{M}\left(\frac{d T}{d \epsilon}\right)} d \epsilon=\int_{0}^{\epsilon_{p}} C(\epsilon) d \epsilon .
$$

For a filament impacted longitudinally at one end, $W$ is the same as the impact velocity.

In deriving the above equations, von Karman assumed a tension-strain curve that was always concave downward. Tension-strain curves for textile yarns often have a concave upward portion which, however, is less pronounced in curves obtained at rapid testing rates. In addition, textile yarns exhibit creep and stress-relaxation phenomena. Such phenomena were not considered by von Karman. Despite this, von Karman's treatment represents a notable advance over theories based on Hookean tension-strain behavior.

\section{Theory of Transverse Impact}

In the laboratory or observer's coordinate system, let $x^{\prime}$ and $y^{\prime}$ denote the horizontal and vertical coordinates, respectively. Let a filament lying along the $x^{\prime}$ axis be impacted with velocity $V$ in the $y^{\prime}$ direction at the point where $x^{\prime}=0$. The motion of the filament is to be described in terms of Lagrangian coordinates. That is, distances along the unstrained length of the filament are given in an $x$ coordinate system, which before impact coincides with the $x^{\prime}$ coordinates. The displacement of any point $x$ of the filament at any time $t$ relative to its original position is given by $\xi(x, t)$ for horizontal displacements and $\eta(x, t)$ for vertical displacements. The location in the laboratory coordinate system of a point $x$ on the filament is thus given by

$$
\begin{aligned}
& x^{\prime}=x+\xi(x, t), \\
& y^{\prime}=\eta(x, t) .
\end{aligned}
$$

2 The terms "elastic" and "plastic" are used as convenient designations for the wave fronts bounding the region of variable strain. They are not intended to characterize the complex recovery behavior of the material. For example, the theory holds for materials having nonlinear tensionstrain curves, but which recover almost completely from large deformations. For these materials, the strain associated with the plastic wave is almost entirely elastic. 
Consider an element of the filament of unstrained length $\Delta x$. After impact, this element is strained, and its new length, measured in the laboratory coordinate system, becomes $\Delta x \sqrt{[1+(\partial \xi / \partial x)]^{2}+(\partial \eta / \partial x)^{2}}$. The increase in length per unit length, or strain $\epsilon$, is thus

$$
\boldsymbol{\epsilon}=\sqrt{\left(1+\frac{\partial \xi}{\partial x}\right)^{2}+\left(\frac{\partial \eta}{\partial x}\right)^{2}}-1 .
$$

The angle $\theta$ that the element makes with the horizontal is given by

$$
\begin{aligned}
& \sin \theta=\frac{1}{1+\epsilon} \frac{\partial \eta}{\partial x} \\
& \cos \theta=\frac{1}{1+\epsilon}\left(1+\frac{\partial \xi}{\partial x}\right) .
\end{aligned}
$$

The differential equations that govern the motion of the small element are

$$
\begin{aligned}
& M \frac{\partial^{2} \eta}{\partial t^{2}}=\frac{\partial}{\partial x}(T \sin \theta), \\
& M \frac{\partial^{2} \xi}{\partial t^{2}}=\frac{\partial}{\partial x}(T \cos \theta),
\end{aligned}
$$

where $M$ is the mass per unit length of the unstrained filament, and $T$ is the tension. By making use of (6a) and (6b), these equations become

$$
\begin{aligned}
& \frac{\partial^{2} \eta}{\partial t^{2}}=\frac{\partial}{\partial x}\left[\frac{T}{M(1+\epsilon)} \frac{\partial \eta}{\partial x}\right], \\
& \frac{\partial^{2} \xi}{\partial t^{2}}=\frac{\partial}{\partial x}\left[\frac{T}{M(1+\epsilon)}\left(1+\frac{\partial \xi}{\partial x}\right)\right] .
\end{aligned}
$$

Assume, as von Karman did, that the tension $T$ is a function $T(\epsilon)$ of the strain only, given by the tension-strain curve. For solutions in which the strain, and consequently the tension, are constant along the filament, eq (8a) and (8b) reduce to

$$
\begin{aligned}
& \frac{\partial^{2} \eta}{\partial t^{2}}=\frac{T}{M(1+\epsilon)} \frac{\partial^{2} \eta}{\partial x^{2}}, \\
& \frac{\partial^{2} \xi}{\partial t^{2}}=\frac{T}{M(1+\epsilon)} \frac{\partial^{2} \xi}{\partial x^{2}}
\end{aligned}
$$

$\xi$ and $\eta$ must also satisfy eq (5).

For other solutions in which $\eta=0$ (no transverse wave), eq (8a), (8b), and (5) reduce to

$$
\begin{gathered}
\eta=0, \\
\frac{\partial^{2} \xi}{\partial t^{2}}=\frac{1}{M} \frac{\partial T}{\partial x}, \\
\epsilon=\frac{\partial \xi}{\partial x} .
\end{gathered}
$$

This is the case considered by von Karman, and to which his solution, eq (1), (2), and (3), is applicable.

A combination of solutions for the preceding special cases gives the solution to the problem in which a long filament initially at rest along an axis in the $x^{\prime}$ direction is impacted transversely at $x=0$ with velocity $V$. The boundary conditions that must be satisfied for the $x>0$ half of the filament are $\xi=0$ and $\eta=V t$ at $x=0$, and $\xi=\eta=0$ at $x=\infty$. 


\section{A Simple Solution Usually Applicable}

Immediately after impact a train of longitudinal-strain waves propagates outward along: the filament. The leading wavelet in the train, or elastic wave, propagates at velocity $C_{e}$ given by eq (1). At points in advance of the elastic wave the filament is unstrained. The elastic wave is succeeded by a series of other more slowly propagating wavelets, each wavelet adding an increment to the strain in the filament. In the wake of each wavelet, material of the filament flows inward toward the point of impact at a velocity that increases as each wavelet passes. The velocity of each wavelet is proportional to the square root of the slope of the tension-strain curve, hence in order that the wavelets do not overtake each other, the slope of the tension-strain curve must decrease as $\epsilon$ increases (be concave downward). The final wavelet in the train, or plastic wave, propagates with velocity $C_{p}$ given by eq (2). The strain in the wake of the plastic-wave front has the constant value $\boldsymbol{\epsilon}_{p}$. Filament material in the wake of the plastic-wave front flows inward at the constant velocity $W$ given by eq (3).

For the simple solution discussed here, transverse-wave motion of the filament occurs only in the wake of the plastic-wave front. The transverse wave thus propagates with velocity $U$, which is less than $C_{p}$. For the region in advance of the point $U t$ on the filament, where only longitudinal-strain waves occur, von Karman's solution [10] is applicable. This solution, satisfying the differential eq (10) and the boundary conditions $\xi=-W t$ at $x=U t$ and $\xi=0$ at $x=\infty$, may be written as follows:

For the region $C_{e} t<x<\infty$,

For the region $C_{p} t \leq x \leq C_{e} t$,

$$
\xi=\eta=\epsilon=0 .
$$

$$
\eta=0 \text {. }
$$

To find the amount of motion in the $\xi$ direction, consider a wavelet, which increases the strain in the filament by an increment $d \epsilon$. This wavelet propagates at velocity $C(\boldsymbol{\epsilon})$ and arrives at point $x$ at time $t=x / C(\epsilon)$. The inward velocity of material flow is increased by an amount $-C(\epsilon) d \epsilon$ during time $t-x / C(\epsilon)$. The distance $\xi$ that point $x$ moves inward is the integrated product of the velocity and time after arrival for each wavelet passing through the point $x$, or

where

$$
\xi=-\int_{0}^{\epsilon} C(\alpha)\left[t-\frac{x}{C(\alpha)}\right] d \alpha=-W(\epsilon) t+\epsilon x
$$

$$
\begin{aligned}
& C(\alpha)=\sqrt{\frac{1}{M}\left(\frac{d T}{d \alpha}\right)}, \\
& W(\epsilon)=\int_{0}^{\epsilon} C^{\gamma}(\alpha(d \alpha),
\end{aligned}
$$

and $\alpha$ is a variable designating strains along the filament in advance of the point where the strain is $\epsilon$.

During the time interval between the arrivals of the elastic and plastic waves at point $x$, the strain is found as a function of $x$ and $t$ by solving the following equation for $\epsilon$ :

For the region $U t \leq x \leq C_{p} t$,

$$
C(\epsilon)=x / t .
$$

$$
\begin{aligned}
& \eta=0, \\
& \xi=-\int_{0}^{\epsilon_{p}} C(\alpha)\left[t-\frac{x}{C(\alpha)}\right] d \alpha=-W t+\epsilon_{p} x, \\
& \epsilon=\epsilon_{p}=\text { a constant. }
\end{aligned}
$$

In order to describe the motion in the transverse-wave region $(0 \leq x \leq U t)$ it is necessary to find solutions of eq (9a), (9b), and (5), which satisfy the appropriate boundary conditions. The most general solution of (9a) has the form $\eta=f+g+A t+B x+K$, where $f$ and $g$ are nonlinear functions of the variables $t-x / \sqrt{T / M(1+\epsilon)}$ and $t+x / \sqrt{T / M(1+\epsilon)}$; respectively. Applying the boundary condition that $\eta \neq V t$ at $x=0$. gives $V t=f(0, t)+g(0, t)+A t+K$. In order that 
this relation be satisfied, it is necessary that $K=0$ and $g(0, t)=-f(0, t)$. Therefore, $f$ and $g$ must be the same function except for sign, and

$$
\eta=f(t-x / \sqrt{T / M(1+\epsilon)})-f(t+x / \sqrt{T / M(1+\epsilon)})+V t+B x .
$$

The condition that $\eta=0$ at $x=U t$ can only be satisfied if $f \equiv 0$ and $B=-V / U$. Thus $\eta=V(t-x / U)$. For this value of $\eta$ and for a constant value of $\epsilon$, the most general solution of eq (5) becomes $\xi=x\left[\sqrt{(1+\epsilon)^{2}-\left(V / U^{2}\right.}-1\right]+h(t)+K$. The condition $\xi=0$ at $x=0$ requires that $h(t)+K=0$. The solution for $\xi$ just obtained is also a solution of eq (9b); thus: For the region $0 \leq x \leq U t$

$$
\begin{aligned}
& \eta=V(t-x / U), \\
& \xi=x\left[\sqrt{\left(1+\epsilon_{2}\right)^{2}-(V / U)^{2}}-1\right], \\
& \epsilon=\epsilon_{2}=\text { a constant. }
\end{aligned}
$$

The behavior of the negative half of the filament is the same as that just described except for the direction of wave propagation.

The configuration of the positive half of the filament at time $t$ after impact is shown in figure 2 by the line passing through the points $A, B, P, Q$. Filament material in the transversewave region $0 \leq x \leq U t$, moves in the $y^{\prime}$ direction because $\partial \xi / \partial t=0$ in this region. Thus points $A, B, P$ moving with velocity $V$, arrive at positions $A^{*}, B^{*}, P^{*}$ at the time $t+d t$. The transversewave front moves with velocity $U$ relative to points on the unstrained filament. The horizontal component of the velocity relative to the $x^{\prime}, y^{\prime}$ coordinate system therefore is $U\left(1+\epsilon_{2}\right) \cos \theta$, and the wave front at point $P$ advances a distance $U d t\left(1+\epsilon_{2}\right) \cos \theta$ to point $Q^{*}$ in a time $d t$. Filament material in advance of the transverse-wave front is moving inward with velocity $W$, so that in time $d t$ point $Q$ moves a distance - $W d t$ to its new position $Q^{*}$.

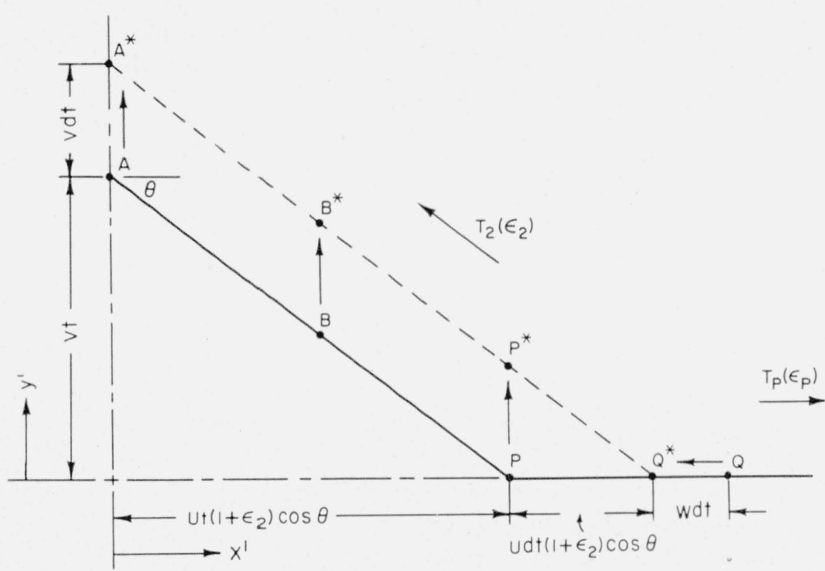

Figure 2. Configuration of positive half of filament $t$ seconds after impact.

Filament originally lay along the $x^{\prime}$ axis and was struck with velocity $V$ in the $y^{\prime}$ direction. After $t$ seconds the positive half of the filament has the configuration $A B P Q$, after $t+d t$ seconds the configuration $A^{*} B^{*} P^{*} Q^{*}$. The filament is assumed to have strain $\epsilon_{2}$ and tension $T_{2}$ in the transverse-wave region $A B P-A^{*} B^{*} Q^{*}$ and strain $\epsilon_{p}$ and tension $T_{p}$ in the region in advance of the transverse wave. (It is proved in the text that in most cases $\epsilon_{2}=\epsilon_{p}, T_{2}=T_{p}$.) Filament material in the transverse wave moves vertically with velocity $V ;$ material in advance of the transverse wave moves inward horizontally with velocity $W$. The transversewave front moves with velocity $U$ relative to points on the unstrained filament but the horizontal component of its velocity relative to the $x^{\prime}, y^{\prime}$ coordinate system is $U\left(1+\epsilon_{2}\right) \cos \theta$. The point $Q$ on the filament moves inward to position $Q^{*}$, the location of the transverse-wave front at time $t+d t$.

The angle $\theta$ that the transverse wave makes with the horizontal is constant along the filament because $\partial \xi / \partial x$ and $\partial \eta / \partial x$ are constant in the region $0 \leq x \leq U t$. The transverse wave is therefore a straight line. $\theta$ is given by either of the relations

$$
\begin{aligned}
& \sin \theta=-\frac{1}{1+\epsilon_{2}} \frac{V}{U}, \\
& \cos \theta=\frac{1}{1+\epsilon_{2}} \sqrt{\left.1+\epsilon_{2}\right)^{2}-\left(\frac{V}{U}\right)^{2}}
\end{aligned}
$$

found by substituting the solutions (14) into the relations (6). 
At the point $x=U t$ where solutions (13) and (14) join, $\xi$ and $\eta$ must be continuous. Continuity of $\eta$ is satisfied because $\eta=0$ for both solutions. Continuity of $\xi$ requires that

$$
U\left[\sqrt{\left(1+\epsilon_{2}\right)^{2}-(V / U)^{2}}-1\right]=\epsilon_{p} U-W .
$$

Horizontal and vertical components of the force must also be continuous. Equating the horizontal components gives

$$
T_{p}-M U W=T_{2} \cos \theta=\frac{T_{2}}{1+\epsilon_{2}} \sqrt{\left(1+\epsilon_{2}\right)^{2}-(V / U)^{2}} .
$$

$M U$ is the element of mass over which the transverse-wave front passes in unit time, so $M U W$ is a force equal to the change of longitudinal momentum per unit time.

Equating the vertical components gives

$$
M U V=-T_{2} \sin \theta=\frac{T_{2}}{1+\epsilon_{2}} \frac{V}{U} .
$$

The velocity of propagation of the transverse wave $U$ is found from eq (18) to be

$$
U=\sqrt{\frac{T_{2}}{M\left(1+\epsilon_{2}\right)}} \cdot
$$

By appropriate manipulation of eq (16), (17), and (18) it is possible to obtain the relation

$$
\frac{T_{p}}{1+\epsilon_{p}}=\frac{T_{2}}{1+\epsilon_{2}} .
$$

The meaning of this relationship is illustrated in figure 3 , where a typical tension-strain curve is

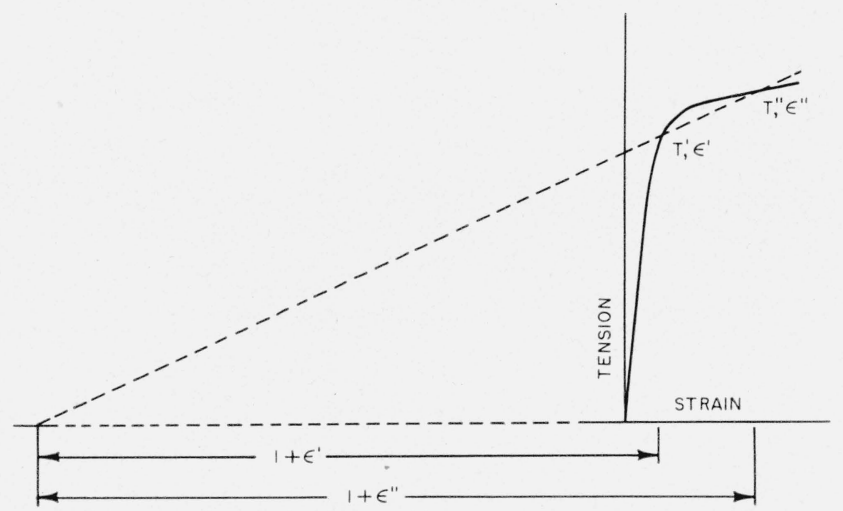

FIGURE 3. Tension-strain curve whose slope decreases to a value less than $T /(1+\epsilon)$.

plotted. The long line extending from the point -1 on the strain axis up to and through any point $\epsilon, T$ on the tension-strain curve has the slope $T /(1+\epsilon)$. The only values of $T$ and $\epsilon$ satisfying eq (20) must be given by intersections of this line with the tension-strain curve. In most cases of practical interest only one intersection exists, and the relation (20) can then be satisfied by

$$
\begin{gathered}
\epsilon_{p}=\epsilon_{2}, \\
T_{p}\left(\epsilon_{p}\right)=T_{2}\left(\epsilon_{2}\right) .
\end{gathered}
$$

Suppose, however, that the tension-strain curve bends over as shown in figure 3 until its slope for large values of $\epsilon$ diminishes to or becomes less than $T /(1+\epsilon)$. A second intersection 
is possible and two sets of $\epsilon, T$ values would be obtained satisfying (20). If a material having such a tension-strain curve were impacted transversely at low speeds, small values of $\epsilon$ and $T$ in the wake of the plastic-wave front would be obtained. The slope of the tension-strain curve for these low values of $\epsilon$ is greater than $T /(1+\epsilon)$, so the plastic-wave front would travel faster than $\sqrt{T_{p} / M\left(1+\epsilon_{p}\right)}$, the velocity $U$ of the transverse-wave front. At a higher impact velocity a strain $\epsilon_{1}$ is obtained at which the slope of the tension-strain curve is just equal to $T_{1} /\left(1+\epsilon_{1}\right)$. In this special case the plastic-wave front and the transverse-wave front are at the same position and move together at the same velocity. If the material is impacted at a higher velocity than this, the solution just given no longer applies. Thus a criterion for the applicability of the simple solution is

$$
\frac{d T}{d \epsilon} \geq \frac{T}{1+\epsilon}
$$

That is, the solution applies if the slope of the tension-strain curve for the material is not less than $T /(1+\epsilon)$ for all strains less than the maximum strain $\epsilon_{p}$, obtained as a result of the impact. Within this restriction the relations (21) can be used to obtain the results given in the following section. A more general solution, which includes the simple solution as a special case, is given later in the text.

\section{Recapitulation of the Simple Solution}

If the tension-strain curve for the filament material obtained at rapid strain rates is always concave downward, if the material does not exhibit large stress relaxation or creep effects during a small time interval, and if the conditions of the impact are such that relation (22) applies, the behavior of a flexible filament after transverse impact is described as follows:

Immediately after transverse impact, longitudinal-strain wavelets propagate outward along the filament. The outermost wavelet, called the elastic wave by von Karman, propagates at velocity

$$
C_{e}=\sqrt{\frac{1}{M}\left(\frac{d T}{d \epsilon}\right)_{\epsilon=0}} .
$$

The innermost, called the plastic wave by von Karman, propagates at velocity

$$
C_{p}=\sqrt{\frac{1}{M}\left(\frac{d T}{d \epsilon}\right)_{\epsilon=\epsilon_{p}} .}
$$

In the region between the elastic- and the plastic-wave fronts the strain increases to $\epsilon_{p}$, the maximum value obtained as a result of the impact. The strain remains constant at this value in the wake of the plastic-wave front.

As the strain wavelets pass a given point on the filament, material of the filament flows inward toward the impact point at a velocity that increases to the value

$$
W=\int_{0}^{\epsilon_{p}} \sqrt{\frac{1}{M} \frac{d T}{d \alpha}} d \alpha
$$

the flow velocity for material in the wake of the plastic-wave front.

The material in the wake of the plastic-wave front forms itself into a transverse wave, shaped like a tent with the impact point at the vertex. The base of the tent spreads outward with velocity

$$
U=\sqrt{\frac{T_{p}}{M\left(1+\epsilon_{p}\right)}} \cdot
$$

It should be noted that the velocity $U$ is with respect to a Lagrangian coordinate system; i. e., a system fixed to the filament, moving and extending with it. Inasmuch as filament mate- 
rail in the wake of the plastic-wave front is in a strained state and moving, the velocity of the transverse-wave front along the filament as seen by an observer in the laboratory would be $\left(1+\epsilon_{p}\right) U-W$. Similarly, the plastic-wave-front velocity in laboratory coordinates would be $\left(1+\epsilon_{p}\right) C_{p}-W$. The elastic-wave front propagates in an unstrained motionless medium and so would have the same velocity in both coordinate systems.

Material in the transverse-wave tent does not move in the horizontal direction but only in the direction of the transverse impact. The velocity $V$ of this motion can be expressed in terms of $\epsilon_{p}, U$, and $W$ by rearranging (16) to obtain

$$
V=\sqrt{\left(1+\epsilon_{p}\right)^{2} U^{2}-\left[\left(1+\epsilon_{p}\right) U-W\right]^{2}} .
$$

If the tension-strain curve for the material is known, it is possible to calculate tables of values for $U, V$, and $W$ as functions of $\epsilon_{p}$. Then, for instance, if the impact velocity is known, it is possible to find the resulting strain $\epsilon_{p}$ and thus find the corresponding values of $U$ and $W$.

\section{General Solution}

When the impact velocity is very great, the transverse-wave front in some cases propagates more rapidly than the plastic-wave front. A solution is then required which provides for a variable strain in the transverse-wave region.

Consider a tension-strain curve that is always concave downward and whose slope eventually becomes less than $T_{1} /\left(1+\epsilon_{1}\right)$, where $\epsilon_{1}, T_{1}$ is the point on the curve such that $d T / d \epsilon=T /(1+\epsilon)$. Such a curve is shown in figure 4 . In the region where $\epsilon_{1}<\epsilon<\epsilon_{p}$, let this curve be approximated by a number of straight-line segments drawn between the points $\epsilon_{1}, T_{1} ; \epsilon_{2}, T_{2} ; \ldots \epsilon_{p}, T_{p}$. Point $\epsilon_{p}, T_{p}$ represents the maximum strain and tension which are attained in the material as the result of an impact.

For this approximate tension-strain curve, consider a solution such as that depicted in figure 5, in which the transverse wave is composed of a number of straight-line segments. The wave front of the leading segment travels at speed $U_{1}$ and the strain in the segment is $\epsilon_{1}$. Wave fronts of succeeding segments travel at decreasing velocities $U_{2}, U_{3}, \ldots U_{p}$, and the successively increasing strains in these segments are $\epsilon_{2}, \epsilon_{3}, \ldots \epsilon_{p}$. In the region in advance of the transverse wave von Karman's solution applies. Thus:

For the region $C_{e} t<x<\infty$,

$$
\eta=\xi=\epsilon=0
$$

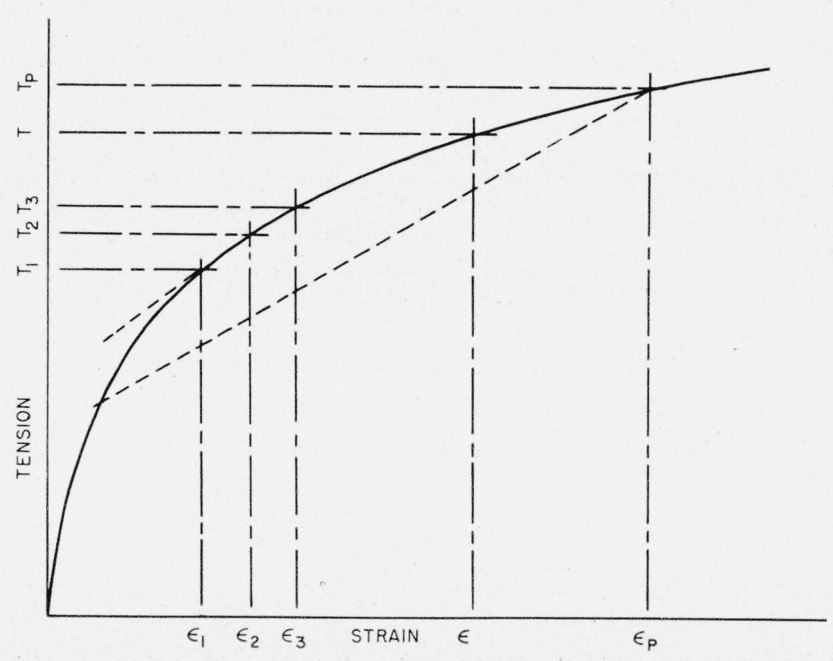

FIgURE 4. Tension-strain curve assumed in derivation of the general solution. 


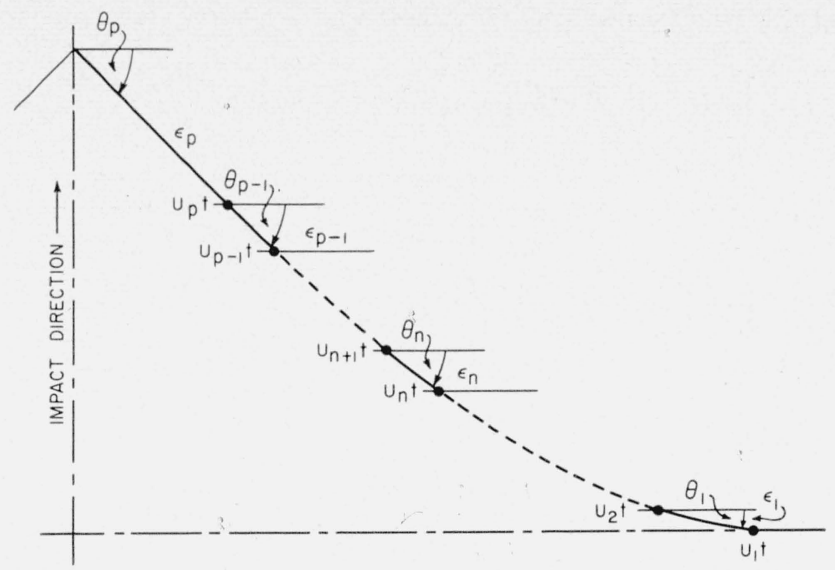

FIGURE 5. Shape of transverse wave assumed in derivation of the general solution.

For the region $U_{1} t \leq x \leq C_{e} t$,

$$
\begin{aligned}
& \eta_{0}=0, \\
& \xi_{0}=-W(\epsilon) t+\epsilon x .
\end{aligned}
$$

In the region of the transverse wave the vertical displacement $\eta_{n}$ in the $n$th segment will have a general solution of the form $\eta_{n}=f_{n}\left(t-x / \sqrt{T / M\left(1+\epsilon_{n}\right)}\right)+g_{n}\left(t+x / \sqrt{T / M\left(1+\epsilon_{n}\right)}\right)+$ $v_{n}\left(t-x / U_{n}\right)+A_{n} t+K_{n}$. The boundary conditions to be satisfied are $\eta_{p}=V t$ at $x=0, \eta_{n}=\eta_{n-1}$ at $x=U_{n} t$ and $\eta_{1}=0$ at $x=U_{1} t$. These boundary conditions require that the $K_{n}=0$ and that $f_{n} \equiv g_{n} \equiv 0$, and provide relations for evaluating the constants $v_{n}$ and $A_{n}$. $\eta_{n}$ thus can be expressed in the form $\eta_{n}=v_{n}\left(t-x / U_{n}\right)+A_{n} t$, which when substituted into (5), gives as a general solution for $\xi_{n}, \xi_{n}=x\left[\sqrt{\left(1+\epsilon_{n}\right)^{2}-\left(v_{n} / U_{n}\right)^{2}}-1\right]+h_{n}(t)+K_{n}$. $\xi_{n}$ is a solution of $(9 \mathrm{~b})$ only when $h(t)$ is a linear function of $t$. Thus $\xi_{n}$ can conveniently be expressed as $\xi_{n}=-w_{n}\left(t-x / U_{n}\right)+$ $B_{n} t+K_{n}$, where $w_{n} / U_{n}=\sqrt{\left(1+\epsilon_{n}\right)^{2}-\left(v_{n} / U_{n}\right)^{2}}-1$. The boundary conditions to be satisfied are $\xi_{p}=0$ at $x=0, \quad \xi_{n}=\xi_{n-1}$ at $x=U_{n} t$, and $\xi_{1}=-W_{1} t+\epsilon_{1} U_{1} t$ at $x=U_{1} t$. These conditions require that the $K_{n}=0$, and provide relations for evaluating the constants $w_{n}$ and $B_{n}$.

The solution for the transverse-wave region thus can be written;

For the interval $U_{2} t \leq x \leq U_{1} t$,

$$
\begin{aligned}
\eta_{1} & =v_{1}\left(t-x / U_{1}\right), \\
\xi_{1} & =-w_{n}\left(t-x / U_{1}\right)-W_{1} t+\epsilon_{1} U_{1} t, \\
w_{1} / U_{1} & =\sqrt{\left(1+\epsilon_{1}\right)^{2}-\left(v_{1} / U_{1}\right)^{2}}-1, \\
\epsilon & =\epsilon_{1} \\
U_{1} & =\sqrt{\frac{1}{M} \frac{T_{1}}{1+\epsilon_{1}}}=\sqrt{\frac{1}{M}\left(\frac{d T}{d \epsilon}\right)_{\epsilon=\epsilon_{1}}} \\
W_{1} & =\int_{0}^{\epsilon_{1}} \sqrt{\frac{1}{M} \frac{d T}{d \alpha}} d \alpha .
\end{aligned}
$$

For the interval $U_{3} t \leq x \leq U_{2} t$,

$$
\begin{aligned}
\eta_{2} & =v_{2}\left(t-x / U_{2}\right)-t\left[v_{1}\left(1-U_{2} / U_{1}\right)\right], \\
\xi_{2} & =-w_{2}\left(t-x / U_{2}\right)-t\left[w_{1}\left(1-U_{2} / U_{1}\right)+W_{1}-\epsilon_{1} U_{1}\right], \\
w_{2} / U_{2} & =\sqrt{\left(1+\epsilon_{2}\right)^{2}-\left(v_{2} / U_{2}\right)^{2}}-1, \\
\epsilon & =\epsilon_{2} .
\end{aligned}
$$


For any interval $U_{n+1} t \leq x \leq U_{n} t$,

$$
\begin{aligned}
\eta_{n} & =v_{n}\left(t-x / U_{n}\right)+t \sum_{k=1}^{k=n-1} v_{k}\left(1-U_{k+1} / U_{k}\right), \\
\xi_{n} & =-w_{n}\left(t-x / U_{n}\right)-t\left[\sum_{k=1}^{k=n-1} w_{k}\left(1-U_{k+1} / U_{k}\right)+W_{1}-\epsilon_{1} U_{1}\right], \\
w_{n} / U_{n} & =\sqrt{\left(1+\epsilon_{n}\right)^{2}-\left(v_{n} / U_{n}\right)^{2}}-1, \\
\boldsymbol{\epsilon} & =\boldsymbol{\epsilon}_{n} .
\end{aligned}
$$

For the interval $0 \leq x \leq U_{p} t_{\text {s }}$

$$
\begin{aligned}
\eta_{p} & =v_{p}\left(t-x / U_{p}\right)+t \sum_{k=1}^{k=p-1} v_{k}\left(1-U_{k+1} / U_{k}\right), \\
\xi_{p} & =-w_{p}\left(t-x / U_{p}\right)-t\left[\sum_{k=1}^{k=p-1} w_{k}\left(1-U_{k+1} / U_{k} !+W_{1}-\epsilon_{1} U_{1}\right],\right. \\
w_{p} / U_{p} & =\sqrt{\left(1+\boldsymbol{\epsilon}_{p}\right)^{2}-\left(v_{p} / U_{p}\right)^{2}}-1, \\
\boldsymbol{\epsilon} & =\boldsymbol{\epsilon}_{p} .
\end{aligned}
$$

A point some distance down the filament thus is motionless until the elastic-wave front reaches it. Subsequently the strain at that point increases, and the point moves inward horizontally at increasing velocity until the tip of the transverse wave reaches it. The strain is then $\epsilon_{1}$ and the inward velocity $W_{1}$. As the first segment of the transverse wave passes through the point the trajectory of the point turns upward in a direction inclined to the vertical. As subsequent elements of higher strain pass through the point the trajectory becomes less inclined, until at strain $\epsilon_{p}$ the direction of travel is vertical, parallel to the impact direction.

The motion of filament material remains vertical in the region $0 \leq x \leq U_{p} t$. There is no horizontal motion of material in this region because of the boundary-condition requirement at $x=0$ that $\xi_{p}=0$. In the region of the strained segments $\epsilon_{1} \leq \epsilon \leq \epsilon_{p}$, some flow of material takes place along the horizontal direction. It is this flow that causes the trajectory of a filament point to be inclined from the vertical. The vertical and horizontal components of velocity of a filament point in this region are given by the coefficients of $t$ in eq (29a) and (29b).

Although for generality each of the strained segments $\epsilon_{n}$ shown in figure 5 has been assumed to have a different angle $\theta_{n}$ of inclination to the horizontal, it will be proved that the angles $\theta_{n}$ are all equal and that the configuration of the transverse wave is a straight line.

The displacements $\xi_{n}, \eta_{n}$, and the horizontal and vertical components of the tension are required to be continuous at each of the points $x=U_{1} t, \ldots U_{n} t, \ldots U_{p} t$. At the point $x=U_{1} t$, $\xi_{1}$ and $\eta_{1}$ are continuous by definition, and continuity of the horizontal and vertical tension components leads to eq $(27 \mathrm{~d})$ and (27e). At $x=U_{n} t$ and at $x=U_{p} t, \xi_{n}$ and $\eta_{n}$ are continuous by definition. Equating horizontal tension components gives

$$
\begin{array}{r}
\frac{T_{n}}{1+\epsilon_{n}} \sqrt{\left(1+\epsilon_{n}\right)^{2}-\left(\frac{v_{n}}{U_{n}}\right)^{2}-M U_{n}\left[w_{n}+\sum_{k=1}^{k=n-1} w_{k}\left(1-\frac{U_{k+1}}{U_{k}}\right)+w_{1}-\epsilon_{1} U_{1}\right]} \\
=\frac{T_{n-1}}{1+\epsilon_{n-1}} \sqrt{\left(1+\epsilon_{n-1}\right)^{2}-\left(\frac{v_{n-1}}{U_{n-1}}\right)^{2}}-M U_{n}\left[w_{n-1}+\sum_{k=1}^{k=n-2} w_{k}\left(1-\frac{U_{k+1}}{U_{k}}\right)+w_{1}-\epsilon_{1} U_{1}\right]
\end{array}
$$

or with the help of eq $(29 \mathrm{c})$

$$
\left[\frac{T_{n}}{1+\epsilon_{n}}-M U_{n}^{2}\right] \sqrt{\left(1+\epsilon_{n}\right)^{2}-\left(\frac{v_{n}}{U_{n}}\right)^{2}}=\left[\frac{T_{n-1}}{1+\epsilon_{n-1}}-M U_{n}^{2}\right] \sqrt{\left(1+\epsilon_{n-1}\right)^{2}-\left(\frac{v_{n-1}}{U_{n-1}}\right)^{2}} .
$$


Equating vertical tension components gives

$$
\frac{T_{n}}{1+\epsilon_{n}} \frac{v_{n}}{U_{n}}-M U_{n}\left[v_{n}+\sum_{k=1}^{k=n-1} v_{k}\left(1-\frac{U_{k+1}}{U_{k}}\right)\right]=\frac{T_{n-1}}{1+\epsilon_{n-1}} \frac{v_{n-1}}{U_{n-1}}-M U_{n}\left[v_{n-1}+\sum_{k=1}^{k=n-1} v_{k}\left(1-\frac{U_{k-1}}{U_{k}}\right)\right]
$$

or

$$
\left[\frac{T_{n}}{1+\epsilon_{n}}-M U_{n}^{2}\right] \frac{v_{n}}{U_{n}}=\left[\frac{T_{n-1}}{1+\epsilon_{n-1}}-M U_{n}^{2}\right] \frac{v_{n-1}}{U_{n-1}}
$$

Manipulation of eq (31) and (32) gives the relation

which reduces to

$$
\left[\frac{T_{n}}{1+\epsilon_{n}}-M U_{n}^{2}\right]\left(1+\epsilon_{n}\right)=\left[\frac{T_{n-1}}{1+\epsilon_{n-1}}-M U_{n}^{2}\right]\left(1+\epsilon_{n-1}\right),
$$

$$
U_{n}=\sqrt{\frac{1}{M} \frac{\left(T_{n}-T_{n-1}\right)}{\left(\epsilon_{n}-\epsilon_{n-1}\right)}}
$$

Equation (34) also holds when $n=p$. Dividing (32) by (33) gives a simple relationhip

$$
\frac{v_{n}}{\left(1+\epsilon_{n}\right) U_{n}}=\frac{v_{n-1}}{\left(1+\epsilon_{n-1}\right) U_{n-1}} .
$$

This relationship also holds when $n=p$. Using (6a), eq (35) becomes

$$
\frac{v_{n}}{\left(1+\epsilon_{n}\right) U_{n}}=\frac{v_{p}}{\left(1+\epsilon_{p}\right) U_{p}}=-\sin \theta_{p}
$$

Applying (36) to (30c), (29b), and (29c), and using (6b), gives the relation

$$
\frac{1}{1+\epsilon_{n}}\left(1+\frac{w_{n}}{U_{n}}\right)=\frac{1}{1+\epsilon_{p}}\left(1+\frac{w_{p}}{U_{p}}\right)=\frac{1}{1+\epsilon_{p}} \sqrt{\left(1+\epsilon_{p}\right)^{2}-\left(\frac{v_{p}}{U_{p}}\right)^{2}}=\cos \theta_{n} .
$$

At $x=0$ the boundary conditions $\eta_{p}=V t$ and $\xi_{p}=0$ must be satisfied. The first of these conditions gives

$$
\begin{aligned}
V=v_{p}+\sum_{k=1}^{k=p-1} \frac{v_{k}}{U_{k}}\left(U_{k}-U_{k-1}\right)=v_{p}+\frac{v_{p}}{\left(1+\epsilon_{p}\right) U_{p}} & \sum_{k=1}^{k=p-1}\left(1+\boldsymbol{\epsilon}_{k}\right)\left(U_{k}-U_{k+1}\right) \\
= & \frac{v_{p}}{\left(1+\boldsymbol{\epsilon}_{p}\right) U_{p}}\left[\sum_{k=1}^{k=p-1} U_{k+1}\left(\boldsymbol{\epsilon}_{k+1}-\boldsymbol{\epsilon}_{k}\right)+\left(1+\boldsymbol{\epsilon}_{1}\right) U_{1}\right] .
\end{aligned}
$$

By the use of (38), eq (36) can be expressed as

$$
\frac{v_{n}}{\left(1+\boldsymbol{\epsilon}_{n}\right) U_{n}}=\frac{V}{\Sigma+\left(1+\boldsymbol{\epsilon}_{1}\right) U_{1}}=-\sin \theta_{n},
$$

where $\Sigma$ is an abbreviation for

Similarly, eq (37) becomes

$$
\Sigma=\sum_{k=1}^{k=p-1} U_{k+1}\left(\epsilon_{k+1}-\epsilon_{k}\right)
$$

$$
\frac{1}{1+\epsilon_{n}}\left(1+\frac{w_{n}}{U_{n}}\right)=\sqrt{1-\left[\frac{V}{\Sigma+\left(1+\epsilon_{1}\right) U_{1}}\right]^{2}}=\cos \theta_{n}
$$

Equation (39) is equivalent to a statement that each $\sin \theta_{n}$ has the same value given by

$$
\sin \theta_{n}=-\frac{V}{\Sigma+\left(1+\epsilon_{1}\right) U_{1}}=\sin \theta .
$$


Similarly, eq (41) states that each $\cos \theta_{n}$ has the same value given by

$$
\cos \theta_{n}=\sqrt{1-\left[\frac{V}{\Sigma+\left(1+\epsilon_{1}\right) U_{1}}\right]^{2}}=\cos \theta .
$$

The configuration of the transverse wave, therefore, is a straight line.

The boundary condition $\xi_{p}=0$ requires that

$$
w_{p}+\sum_{k=1}^{k=p-1} w_{k}\left(1-\frac{U_{k+1}}{U_{k}}\right)+W_{1}-\epsilon_{1} U_{1}=0
$$

It can be shown that

$$
\begin{aligned}
w_{p}+\sum_{k=1}^{k=p-1} w_{k}\left(1-\frac{U_{k+1}}{U_{k}}\right) & =\frac{1}{1+\epsilon_{p}}\left(1+\frac{W_{p}}{U_{p}}\right)\left[\sum_{k=1}^{k=p-1} U_{k+1}\left(\epsilon_{k+1}-\epsilon_{k}\right)+\left(1+\epsilon_{1}\right) U_{1}\right]-U_{1} \\
& =\left[\Sigma+\left(1+\epsilon_{1}\right) U_{1}\right] \cos \theta-U_{1} \\
& =\sqrt{\left[\Sigma+\left(1+\epsilon_{1}\right) U_{1}\right]^{2}-V^{2}}-U_{1}
\end{aligned}
$$

so that eq (44) can be put into the form

$$
V=\sqrt{\left[\Sigma+\left(1+\epsilon_{1}\right) U_{1}\right]^{2}-\left[\left(1+\epsilon_{1}\right) U_{1}-W_{1}\right]^{2}}
$$

Equations (46) and (42) can be combined to obtain two different expressions for $\sin \theta$ and $\cos \theta$

$$
\begin{aligned}
& \sin \theta=-\sqrt{1-\left[\frac{\left(1+\epsilon_{1}\right) U_{1}-W_{1}}{\Sigma+\left(1+\epsilon_{1}\right) U_{1}}\right]^{2}}, \\
& \cos \theta=\frac{\left(1+\epsilon_{1}\right) U_{1}-W_{1}}{\Sigma+\left(1+\epsilon_{1}\right) U_{1}} .
\end{aligned}
$$

Equations (31) through (48) can now be used to modify the solutions given earlier. Thus (30a) and (30b) become

$$
\begin{gathered}
\eta_{p}=\left\{v_{p}+\sum_{k=1}^{k=p-1} v_{k}\left(1-\frac{U_{k+1}}{U_{k}}\right)\right\} t-\frac{v_{p}}{U_{p}} x \\
=V\left\{t-\frac{\left(1+\epsilon_{p}\right) x}{\Sigma+\left(1+\epsilon_{1}\right) U_{1}}\right\} . \\
\xi_{p}=-\left\{w_{p}+\sum_{k=1}^{k=p-1} w_{k}\left(1-\frac{U_{k+1}}{U_{k}}\right)+W_{1}-\epsilon_{1} U_{1}\right\} t+\frac{w_{p}}{U_{p}} x \\
=\left\{\left(1+\epsilon_{p}\right) \cos \theta-1\right\} x=-\left\{1-\left(1+\epsilon_{p}\right)\left[\frac{\left(1+\epsilon_{1}\right) U_{1}-W_{1}}{\Sigma+\left(1+\epsilon_{1}\right) U_{1}}\right]\right\} x .
\end{gathered}
$$

and $(29 \mathrm{a})$ and $(29 \mathrm{~b})$ become

$$
\begin{aligned}
\eta_{n} & =t\left\{v_{n}+\sum_{k=1}^{k=n-1} v_{k}\left(1-\frac{U_{k+1}}{U_{k}}\right)\right\}-\frac{v_{n}}{U_{n}} x \\
& =t\left\{\frac{v_{n}}{\left(1+\epsilon_{n}\right) U_{n}}\left[\sum_{k=1}^{k=n-1} U_{k}\left(\epsilon_{k+1}-\epsilon_{k}\right)+\left(1+\epsilon_{1}\right) U_{1}\right]\right\}-\frac{\left(1+\epsilon_{n}\right) V}{\Sigma+\left(1+\epsilon_{1}\right) U_{1}} x \\
& =V\left\{\left[\frac{\sum_{k=1}^{k=n-1} U_{k}\left(\epsilon_{k+1}-\epsilon_{k}\right)+\left(1+\epsilon_{1}\right) U_{1}}{\sum+\left(1+\epsilon_{1}\right) U_{1}}\right] t-\left[\frac{1+\epsilon_{n}}{\sum+\left(1+\epsilon_{1}\right) U_{1}}\right] x\right\} .
\end{aligned}
$$




$$
\begin{aligned}
\xi_{n} & =-\left\{w_{n}+\sum_{k=1}^{k=n-1} w_{k}\left(1-\frac{U_{k+1}}{U_{k}}\right)+W_{1}-\epsilon_{1} U_{1}\right\} t+\frac{w_{n}}{U_{n}} x \\
& =-\left\{\left[\sum_{k=1}^{k=n-1} U_{k+1}\left(\epsilon_{k+1}-\epsilon_{k}\right)+\left(1+\epsilon_{1}\right) U_{1}\right] \cos \theta-\left[\left(1+\epsilon_{1}\right) U_{1}-W_{1}\right]\right\} t-\left\{1-\left(1+\epsilon_{n}\right) \cos \theta\right\} x \\
& =+\left[\left(1+\epsilon_{1}\right) U_{1}-W_{1}\right]\left\{1-\frac{\sum_{k=1}^{k=n-1} U_{k+1}\left(\epsilon_{k+1}-\epsilon_{k}\right)+\left(1+\epsilon_{1}\right) U_{1}}{\sum+\left(1+\epsilon_{1}\right) U_{1}}\right\} t \\
& -\left\{1-\left(1+\epsilon_{n}\right)\left[\frac{\left(1+\epsilon_{1}\right) U_{1}-W_{1}}{\sum+\left(1+\epsilon_{1}\right) U_{1}}\right]\right\}_{x}
\end{aligned}
$$

The equations just given are for the case in which the tension-strain curve is approximated in the region $\epsilon_{1} \leq \epsilon \leq \epsilon_{p}$ by a finite number of straight-line segments. The solution sought is the limit solution when the number of straight-line segments approaches infinity and the length of each segment approaches zero. This limit solution is given in the following section.

\section{Tabulation and Interpretation of the General Solution}

For the interval $0 \leq x \leq U_{p} t$,

$$
\begin{aligned}
& \eta=V\left\{t-\frac{\left(1+\epsilon_{p}\right) x}{S+\left(1+\epsilon_{1}\right) U_{1}}\right\}, \\
& \xi=-\left\{1-\left(1+\epsilon_{p}\right)\left[\frac{\left(1+\epsilon_{1}\right) U_{1}-W_{1}}{S+\left(1+\epsilon_{1}\right) U_{1}}\right]\right\} x \\
& \epsilon=\epsilon_{p} .
\end{aligned}
$$

For the interval $U_{p} t \leq x \leq U_{1} t$,

$$
\begin{aligned}
& \eta=V\left\{\left[\frac{S(\epsilon)+\left(1+\epsilon_{1}\right) U_{1}}{S+\left(1+\epsilon_{1}\right) U_{1}}\right] t-\left[\frac{1+\epsilon}{S+\left(1+\epsilon_{1}\right) U_{1}}\right] x\right\}, \\
& \xi=\left[\left(1+\epsilon_{1}\right) U_{1}-W_{1}\right]\left\{1-\frac{S(\epsilon)+\left(1+\epsilon_{1}\right) U_{1}}{S+\left(1+\epsilon_{1}\right) U_{1}}\right\} t-\left\{1-(1+\epsilon)\left[\frac{\left(1+\epsilon_{1}\right) U_{1}-W_{1}}{S+\left(1+\epsilon_{1}\right) U_{1}}\right]\right\} x .
\end{aligned}
$$

For the region $U_{1} t \leq x<\infty$ the solution is given by eq (25) and (26). The symbols used in the above equations have been defined previously, except for

and

$$
S=\int_{\epsilon_{1}}^{\epsilon_{p}} \sqrt{\frac{1}{M}} \frac{d T}{d \alpha} d \alpha=W^{\prime}-W_{1}
$$

$$
S(\epsilon)=\int_{\epsilon_{1}}^{\epsilon} \sqrt{\frac{1}{M} \frac{d T}{d \alpha}} d \alpha=W(\epsilon)-W_{1} .
$$

The general solution given in this section differs from the simple solution given previously in that the strain in the transverse wave need not be constant. At low impact speeds the equations of the general solution reduce to those of the simple solution. The transverse wave then propagates in a region of constant strain in the wake of the plastic-wave front. However, for a sufficiently high impact velocity and a tension-strain curve of the right shape, the case in which the transverse-wave front and the plastic-wave front coincide occurs. The velocity of propagation of these two wave fronts is $U_{1}$ and the maximum strain in the filament is $\epsilon_{1}$. If the tension-strain curve is known, the values of $U_{1}$ and $\epsilon_{1}$ for this special case can be found from eq (27e). 
At higher transverse impact-velocities the plastic-wave front propagates more slowly than the transverse-wave front. A region of variable straining therefore occurs in the transverse wave, and the general solution must be used. The shape of the transverse wave remains a straight line, but the propagation velocity no longer increases with increasing impact velocity. It remains constant at the value $U_{1}$. The velocity of propagation of the maximum strained portion of the transverse wave $U_{p}$ is the same as the plastic-wave velocity $C_{p}$ and is computed from eq (2). In order to find the value of the maximum strain, or constant strain $\epsilon_{p}$ in the wake of the plastic-wave front, the relation

$$
V=\sqrt{\left[S+\left(1+\epsilon_{1}\right) U_{1}\right]^{2}-\left[\left(1+\epsilon_{1}\right) U_{1}-W_{1}\right]^{2}}
$$

can be used. The value of $S$ depends upon $\epsilon_{p}$, so that if the tension-strain curve is known, the value of $\epsilon_{p}$ needed to give a required $V$ can be found by a process of trial and error.

At the transverse-wave front, material of the filament is flowing inward horizontally with velocity $W_{1}$. At the point in the transverse wave where the strain attains its maximum value $\epsilon_{p}$, material is flowing inward along the filament direction with velocity $W$. The quantity $S$ represents the increase in velocity over $W_{1}$ due to the strain increase in the transverse-wave region.

The motion of filament points in the wake of the plastic-wave front is most easily visualized as follows; Suppose there is a second filament, inextensible and fixed to the impacted filament at a point a great distance in advance of the elastic-wave front. Let the end of this filament coincide with the impact point. After impact let the filament be constrained to move alongside the impacted filament but without extending so that its end no longer coincides with the impact point, but recedes from it. To an observer at this filament end the impacted filament would appear to have motion with velocity $W$.

Although flow of filament material in the wake of the plastic wave occurs, there is no horizontal component of the flow velocity. A horizontal component of the flow does occur in the region where the strain varies: i. e., where $\epsilon_{1} \leq \epsilon \leq \epsilon_{p}$. The magnitude of this velocity component is much smaller than $W_{1}$, the horizontal flow velocity in advance of the transverse wave, and decreases to zero where $\epsilon$ equals $\epsilon_{p}$.

At low impact speeds, where the simple theory applies, the angle that the transverse wave makes with the horizontal can be found from the equation

$$
\cos \theta=\frac{\left(1+\epsilon_{p}\right) U-W}{\left(1+\epsilon_{p}\right) U}
$$

As the impact velocity is increased, $\cos \theta$ decreases to the value

$$
\cos \theta_{1}=\frac{\left(1+\epsilon_{1}\right) U_{1}-W_{1}}{\left(1+\epsilon_{1}\right) U_{1}} .
$$

( $\theta$ is a negative angle and attains a greater negative value as the impact velocity is increased.) At higher impact speeds, where only the general theory applies, $\theta$ can be found from the relation

$$
\cos \theta=\frac{\left(1+\epsilon_{1}\right) U_{1}-W_{1}}{S+\left(1+\epsilon_{1}\right) U_{1}}
$$

The values of $\epsilon_{1}, U_{1}$, and $W_{1}$ are constants, but $S$ increases as the impact velocity is increased. $\operatorname{Cos} \theta$ continues to decrease, and the value of $\theta$ becomes greater negatively. Thus, although the progagation velocity of the transverse wave is constant for all impact speeds above a certain value, the slope of the transverse wave continues to change.

Figure 6 , a, shows the configuration of the filament after a transverse impact of sufficiently slow speed that the simple theory applies. Figure $6, \mathrm{~b}$, shows the configuration after a higher speed impact where only the general theory applies. As pointed out previously, the formula for $U$ expresses the transverse wave velocity relative to a Lagrangian coordinate system. 

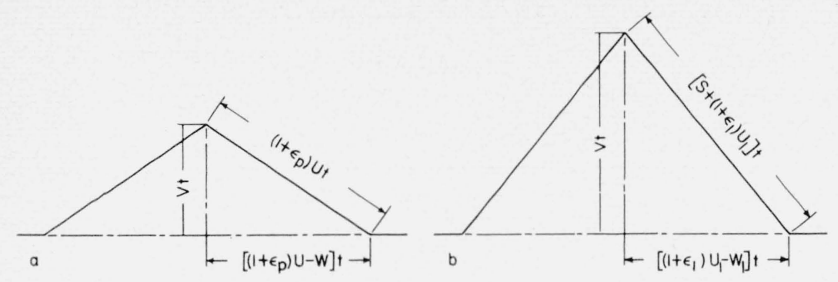
FiguRE 6. Configuration of filament $t$ seconds after transverse impact.
a. Simple theory; b. General theory.

In laboratory coordinates the distance that the transverse wave travels along the filament in unit time is $\left(1+\epsilon_{p}\right) U$ for the simple theory, and $S+\left(1+\epsilon_{1}\right) U_{1}$ for the general theory. The corresponding distances that the wave front travels in the horizontal direction are $\left(1+\epsilon_{p}\right) U-W$ and $\left(1+\epsilon_{1}\right) U_{1}-W_{1}$, respectively. These distances are labeled in figure 6 , a and $\mathrm{b}$. Thus it is seen how some of the relationships derived above could have been obtained very simply from a knowledge of geometry and an intuitive understanding of the problem.

\section{Examples of the Theory}

In order to illustrate the theory, the behavior of a filament after transverse impact is computed for two different hypothetical materials. The first material obeys Hooke's law. Its tension-strain curve is given by

$$
\frac{T}{M}=C^{2} \epsilon
$$

As this tension-strain curve is a straight line of constant slope, the simple theory applies. The elastic- and plastic-wave fronts both propagate with velocity $C$. $U, V$, and $W$ are given by

$$
\begin{aligned}
& U=C \sqrt{\frac{\boldsymbol{\epsilon}_{p}}{1+\boldsymbol{\epsilon}_{p}}}, \\
& V=C \sqrt{\boldsymbol{\epsilon}_{p}\left(1+\boldsymbol{\epsilon}_{p}\right)-\left[\sqrt{\boldsymbol{\epsilon}_{p}\left(1+\boldsymbol{\epsilon}_{p}\right)}-\boldsymbol{\epsilon}_{p}\right]^{2}}, \\
& W=C \boldsymbol{\epsilon}_{p} .
\end{aligned}
$$

Curves of $U / C, V / C$, and $W / C$ as functions of $\epsilon_{p}$ for the Hookean material are plotted in figure 7 . The solution of a transverse-impact problem may be found from these curves by reading off the values or $\epsilon_{p}, U / C$, and $W / C$ corresponding to a particular $V / C$. The value of $C$ is determined from the Young's modulus and density of the unstrained material.

In order to illustrate a case in which the general theory applies, computations were made for a material whose tension-strain curve is given by ${ }^{3}$

$$
\frac{T}{M}=14 \tanh 20 \epsilon \text {. }
$$

For this curve, plotted in figure 8, the units of $T / M$ are arbitrary, and the strain $\epsilon$ is expressed as a fraction of unity. The slope of the curve, initially 280 arbitrary units per unit strain, continually decreases and approaches a value of zero for very large strains. The slope $d(T / M) / d \epsilon$ and the quantity $T / M(1+\epsilon)$ become equal to 12.308 at a strain of 0.11221 . When $\epsilon$ is 0.20 , the highest strain for which computations were made, the slope is 0.37547 . In the region $0 \leq x \leq 0.11221$ the simple theory applies. In the region $0.11221 \leq \epsilon<\infty$ the general theory applies.

The elastic-wave velocity is $C_{e}=\sqrt{280}=16.733$ arbitrary velocity units. The plasticwave velocity is given by

$$
C_{p}=\sqrt{\frac{d}{d \epsilon}\left(\frac{T}{M}\right)_{\epsilon=\epsilon_{p}}}=\sqrt{280 \operatorname{sech}^{2} 20 \epsilon_{p}}=C_{e} \operatorname{sech} 20 \epsilon_{p} .
$$

\footnotetext{
${ }^{3}$ The functional relationship (62) was chosen because tables were available that simplified the calculations. The numbers 14 and 20 have no significance, and were chosen only for convenience in plotting the curve. Other relationships involving more familiar functions could have been used, such as, $T / M=a+b \boldsymbol{\epsilon}-\sqrt{a^{2}+b^{2} \boldsymbol{\epsilon}^{2}}$, where $a$ and $b$ are arbitrary constants.
} 


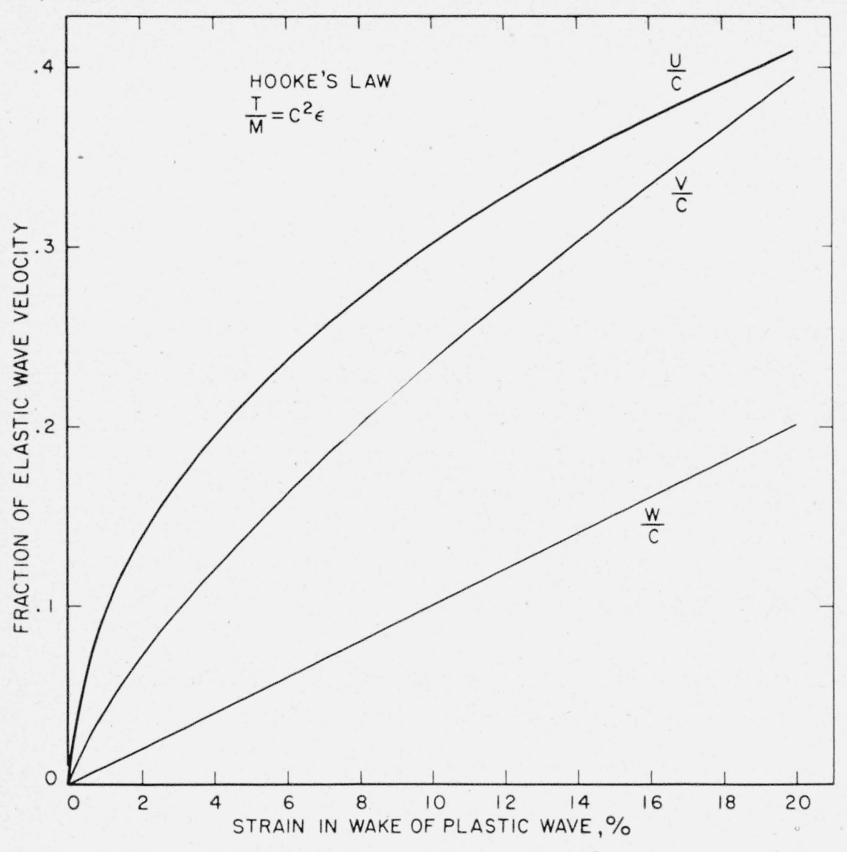

Figure 7. Curves of $U / C, V / C$, and $W / C$ as functions of $\epsilon_{p}$ for a filament having a linear tension-strain curve.

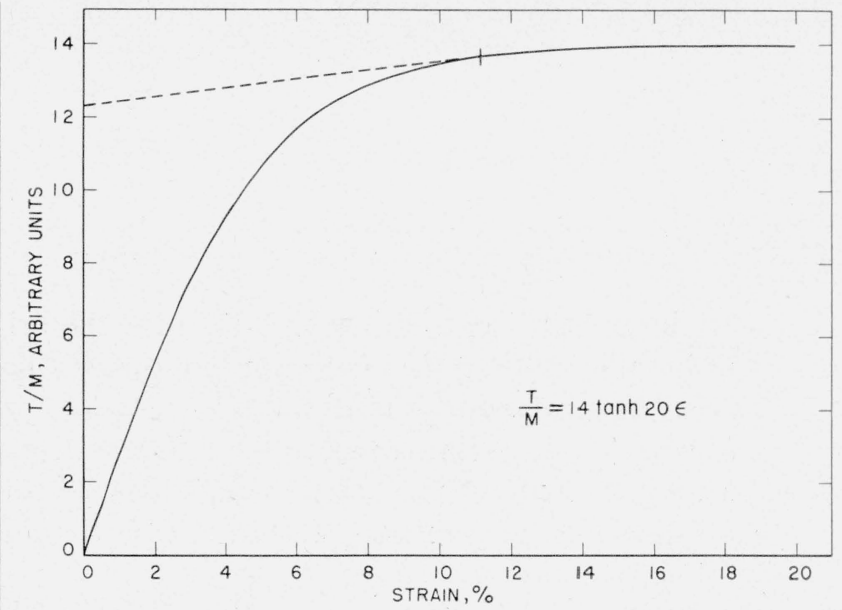

Figure 8. Plot of tension-strain curve given by $(T / M)=14 \tanh 20 \epsilon$.

Point of tangency with dotted line indicates strain and tension at which the slope of the curve equals $T / M(1+\epsilon)$.

In the region $0 \leq \epsilon_{p} \leq 0.11221$ the transverse-wave velocity is given by

$$
U=\sqrt{\frac{T}{M\left(1+\epsilon_{p}\right)}}=C_{e} \sqrt{\frac{0.05 \tanh 20 \epsilon_{p}}{1+\epsilon_{p}}} .
$$

When $\epsilon_{p}$ is equal to or greater than 0.11221 , the transverse-wave velocity is constant at the value $U_{1}=0.20967 C_{e}$.

Filament material in the wake of the plastic-wave front flows inward at a velocity given by

$$
W=\int_{0}^{\epsilon_{p}} \sqrt{\frac{1}{M} \frac{d T}{d \alpha}} d \alpha=0.05 C_{e} \tan ^{-1}\left(\sinh 20 \epsilon_{p}\right) .
$$

Figure 9 gives the curves of $U / C_{e}, V / C_{e}$, and $W / C_{e}$ as functions of $\epsilon_{p}$ for this material. The dotted lines give portions of the corresponding curves for the Hookean material.

Other behavior features of a material having the nonlinear tension-strain curve of eq (62) are given in figures $10,11,12$, and 13 . Figure 10 shows the strain distribution along the filament after transverse impact at various speeds up to $0.177 C_{e}$. The dimensionless parameter $x / C_{e} t$ is used as the abscissa. A particular value of this parameter, 0.6 for instance, can be interpreted as a distance down the fiber 0.6 as far as the elastic wave has propagated. The dotted line gives the strain at the transverse-wave front as a function of the location of the transverse-wave front. Thus the strain in the filament after a transverse impact at velocity of $0.151 C_{e}$ increases as shown up to a strain of 8 percent between the elastic- and plastic-wave fronts, and then remains constant. The plastic-wave front propagates at a velocity of $C_{p}=$ $0.388 C_{e}$, and the transverse-wave front at a velocity of $U=0.207 C_{e}$ for this case.

Figure 11 shows the horizontal component of the inward-flow velocity for different points along the filament. The location of a point on the filament is designated by the same distance parameter used in figure 10. Consider, for instance, the case in which the filament is impacted at velocity $0.173 C_{e}$. The strain in the wake of the plastic-wave front is 16 percent. The flow velocity at various points along the filament between the elastic-wave front and the transverse-wave front at $x / C_{e} t=0.210$ increases to the value $0.0680 C_{e}$ as shown. As the filament is horizontal in this region all of the flow is horizontal and directed inward. In the wake of the transverse-wave front the filament has changed its direction. The horizontal component 


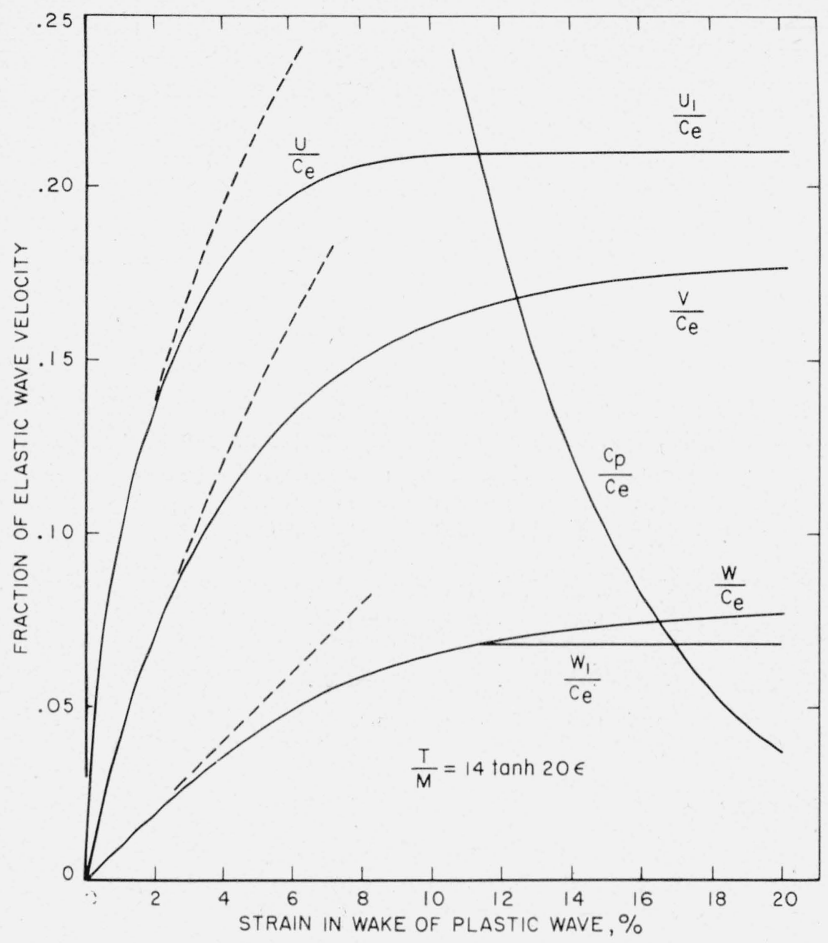

Figure 9. Curves of $U / C_{e}, V / C_{e}$, and $W / C_{e}$ as functions of $\epsilon_{p}$ for a filament with tension-strain curve given by $(T / M)=14 \tanh 20 \epsilon$.

Dotted lines show portions of similar curves for filament having linear stressstrain curve.

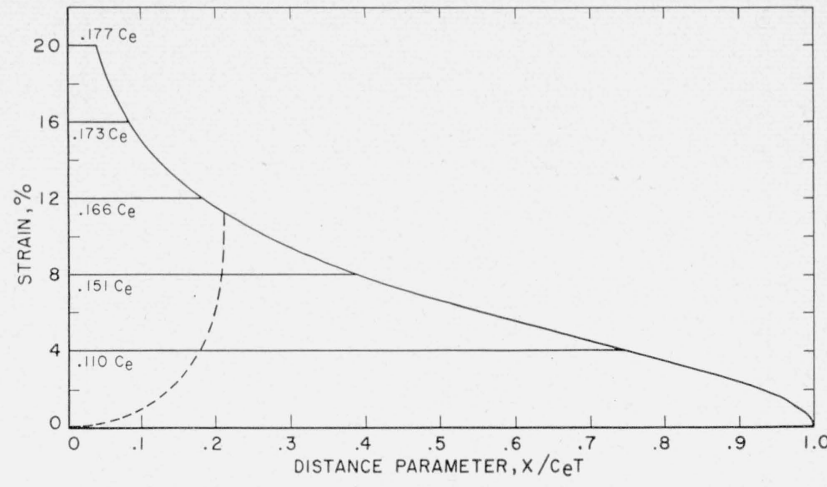

FIGURE 10. Strain distribution along filament having tensionstrain curve given by $(T / M)=14$ tanh $20 \epsilon$, after transverse impact.

If the filament is impacted, for example, with velocity $0.151 \mathrm{C}_{e}$, the strain increases to a value of 8 percent in the region between the elastic-and plastic-wave fronts, and then remains constant. The plastic-wave front in this ease propagates at a velocity of $C_{p}=0.388 C_{e}$, its position along the filament in distance parameter units is thus 0.388 . The position of the dotted line gives the position of the transverse-wave front, which in this case propagates at velocity $U=0.207 C_{\varepsilon}$.

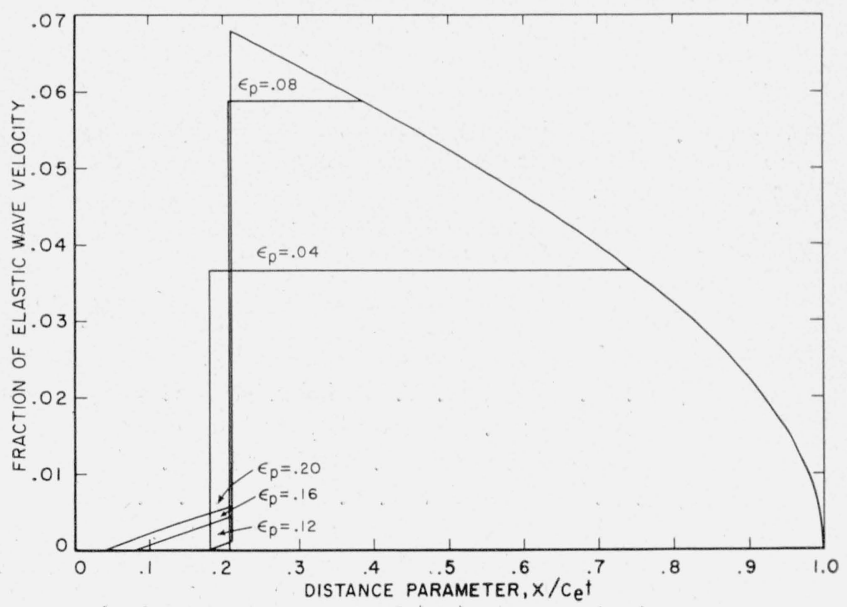

FIGURE 11. Horizontal component of flow velocity at various points along the length of filament having tension-strain curve given by $(T / M)=14$ tanh $20 \epsilon$, after transverse impact.

A filament impacted at velocity $V=0.173 C_{e}$, for example, has a strain in the wake of the plastic-wave front of 16 percent. The inward-flow velocity at various points along the filament between the elastic-wave front and the transverse-wave front at $x / C_{e} t=0.210$ increases to the value $0.0680 C_{e}$. In the wake of the transversewave front the filament has changed direction, and the horizontal component'of flow velocity, therefore, decreases abruptly to the value $0.0045 C_{e}$, and then decreases gradually to zero at $x / C_{e} t=0.081$, the location of the plastic-wave front.

of the flow velocity, therefore, decreases abruptly to the value $0.0045 C_{e}$. It then decreases gradually to zero at $x / C_{e} t=0.081$, the location of the plastic-wave front. Between the plasticwave front and the impact point the horizontal component of the flow velocity is zero.

Figure 12 shows how the angle $\theta$ of the transverse wave varies with the strain $\epsilon_{p}$ in the wake of the plastic-wave front. The solid line gives calculated values of $\cos \theta$ and $\theta$ for the material with the nonlinear tension-strain curve. The dotted line gives the values calculated for the Hookean material. The angle $\theta$ increases negatively very rapidly for small values of $\epsilon_{p}$. Thus even at low values of $\epsilon_{p}$ and correspondingly low impact velocities, a well-defined tent-shaped wave may be expected. (For instance, if $C_{e}=2,400 \mathrm{~m} / \mathrm{sec}$ and $V=0.026 C_{e}=62.5$ $m / \mathrm{sec}, \epsilon_{p}=0.5$ percent and $\theta=-21.75^{\circ}$.) At higher values of $\epsilon_{p}$ the configuration of the transverse wave does not change much. This is illustrated in figure 13, where several configurations after impact are plotted for the filament having the nonlinear stress-strain curve. The locations of the plastic-wave front on several of these configurations where $\epsilon_{p}$ exceeds 0.11221 are given by the black dots. 


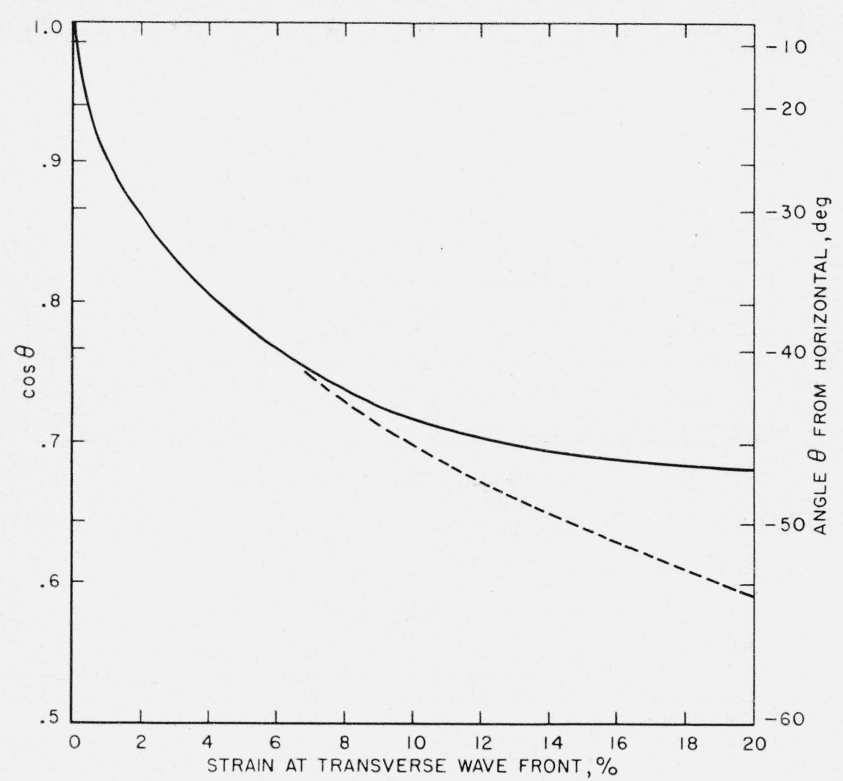

FIGURE 12. Angle $\theta$ which transverse wave makes with horizontal plotted as a function of the strain $\left(\epsilon_{p}\right.$ or $\left.\epsilon_{1}\right)$ at the transverse-wave front.

Solidline: Filament having tension-strain curve given by $(T / M)=14 \tanh 20 \epsilon$. Dotted line: Filament having linear tension-strain curve.

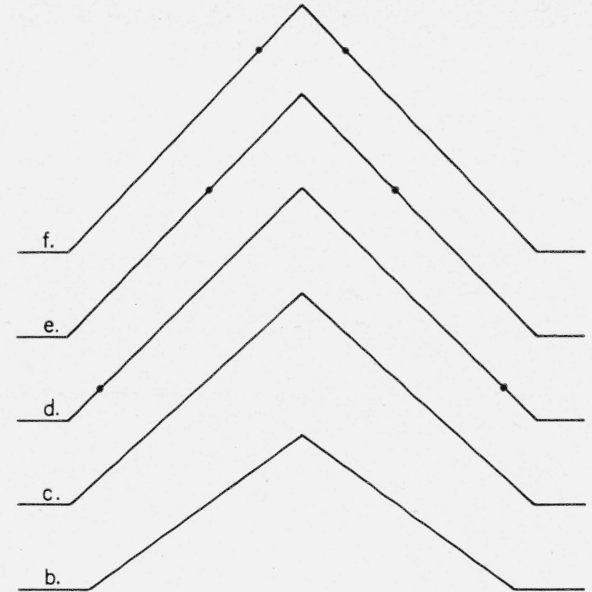

a.

FIgUre 13. Configurations after transverse impact for filament having tension-strain curve given by $(T / M)=14$ tanh $20 \epsilon$.

a. Before impact.

b. $\epsilon_{p}=0.04, V=0.110 C_{e}, \theta=-36.4^{\circ}$.

c. $\epsilon_{p}=.08, V=.151 C_{e}, \theta=-42.5^{\circ}$.

d. $\epsilon_{p}=.12, V=.166 C_{e}, \theta=-45.3^{\circ}$

e. $\epsilon_{p}=.16, V=.173 C_{e}, \theta=-46.4^{\circ}$

f. $\epsilon_{p}=.20, V=.177 C_{e}, \theta=-46.9^{\circ}$.

Black dots in last three configurations show positions of plastie-wave fronts.

\section{References}

[1] W. K. Stone, H. F. Schiefer, and G. Fox, Stress-strain relationships in yarns subjected to rapid impact loading: 1. Equipment, testing procedure, and typical results, J. Research NBS 54, 269 (1955) RP2589; Textile Research J. 25, 520 (1955).

[2] F. L. McCrackin, H. F. Schiefer, J. C. Smith, and W. K. Stone, Stress-strain relationships in yarns subjected to rapid impact loading: 2. Breaking velocities, strain energies, and theory neglecting wave propagation, J. Research NBS 54, 277 (1955) RP2590; Textile Research J. 25, 529 (1955).

[3] J. C. Smith, F. L. McCrackin, and H. F. Schiefer, Stress-strain relationships in yarns subjected to rapid impact loading: 3. Effect of wave propagation, J. Research NBS 55, 19 (1955) RP2601; Textile Research J. 25, 701 (1955).

[4] J. C. Smith, F. L. McCrackin, H. F. Schiefer, W. K. Stone, and K. M. Towne, Stress-strain relationships in yarns subjected to rapid impact loading: 4. Transverse impact tests, J. Research NBS 57, 83 (1956) RP2695; Textile Research J. 26, 821 (1956).

[5] G. I. Taylor, unpublished (1942).

[6] J. D. Cole, C. B. Dougherty, and J. H. Huth, Constant strain waves in strings, J. Appl. Mechanics, 20, 519 (1953).

[7] J. W. Craggs, Wave motion in plastic-elastic strings, J. Mech. and Phys. of Solids 2, 286 (1954).

[8] F. L. McCrackin, Impact tests of textile filaments, Doctor's dissertation, Lehigh University, Bethlehem, Pa. (1957).

[9] F. O. Ringleb, Motion and stress of an elastic cable due to impact, J. Appl. Mechanics 24, 417 (1957).

[10] T. von Karman and P. Duwez, The propagation of plastic deformation in solids, J. Appl. Phys. 21, 987 (1950).

[11] G. I. Taylor, The plastic wave in a wire extended by an impact load, British Official Report R. C. 329 (1942).

Washington, September 19, 1957. 